\title{
Hypopharyngeal perforation: an uncommon cause of pneumoperitoneum
}

\author{
S A A Woodcock, H Bird, A K Siriwardena, S Ellenbogen
}

\begin{abstract}
Endotracheal intubation is common practice being performed both electively and as an emergency. Complications of this procedure are uncommon. A case is described of hypopharyngeal rupture after emergency intubation that presented with a pneumoperitoneum. The clinical signs, relevant investigations and management options are discussed for this injury and the need is emphasised for a high index of suspicion in order to make an early diagnosis.

(Emerg Med f 2001;18:396-398)
\end{abstract}

Keywords: hypopharyngeal perforation; endotracheal intubation

Hypopharyngeal perforation secondary to traumatic endotracheal intubation is a rare but serious complication. Early clinical signs of this condition include subcutaneous emphysema, mediastinal emphysema and pneumothorax. ${ }^{1}$ We describe a clinical case of a hypopharyngeal perforation where the initial findings included a pneumoperitoneum, which to our knowledge has not been previously described.

Department of Surgery, Tameside General Hospital, Ashton under Lyne, Lancashire

S A A Woodcock S Ellenbogen

Department of Anaesthetics, Tameside General Hospital H Bird

\section{Department of} Surgery, Manchester Royal Infirmary, Manchester

A K Siriwardena

Correspondence to: Mr Woodcock, Department of Surgery, Manchester Royal Infirmary, Oxford Road, Manchester M13 9WL, UK

(sean.woodcock@virgin.net)

Accepted for publication 26 September 2000

\section{Case report}

A 16 year old man was witnessed to collapse outside his home. An ambulance arrived approximately 20 minutes after the collapse. The patient was found lying on his back, cyanosed, unconscious and with fixed dilated pupils. Cardiopulmonary resuscitation (CPR) was started and an endotracheal tube passed. There was no report of a difficult intubation. After several minutes the attending ambulance crew noticed swelling of the patient's neck and so removed and then replaced the endotracheal tube. The patient was transferred to the accident and emergency department where CPR continued. The initial ECG indicated electromechanical dissociation and the patient was treated with intravenous adrenaline (epinephrine). The cardiac rhythm changed to ventricular fibrillation and the patient was treated by external D/C shock at $200 \mathrm{~J}$ and 400 $\mathrm{J}$ without success. Chest examination suggested bilateral pneumothoraces therefore large bore cannulas were placed bilaterally into the second rib space in the midclavicular line with an associated bilateral release of air. The patient reverted to sinus rhythm with a heart rate of 150 beats per minute supporting a blood pressure of 130/65 $\mathrm{mm} \mathrm{Hg}$.

On further examination the pupils were still fixed and dilated. A small abrasion was noted on the scalp. There was marked surgical emphysema extending from the face to the umbilicus. The trachea was central, chest expansion and breath sounds equal bilaterally. Examination of the abdomen revealed marked distension with increased resonance and an absence of bowel sounds. Examination of the back was unremarkable.

Chest radiography revealed marked surgical emphysema in the subcutaneous tissues and mediastinum, bilateral small pneumothoraces and the double wall sign of abdominal viscera suggestive of free air in the peritoneum

A nasogastric tube was passed, chest drains inserted and intravenous Co-Amoxyclav and ranitidine administered. A lateral decubitus film confirmed the presence of free air in the abdominal cavity (fig 2). The diagnosis of a pharyngo-oesophageal perforation was

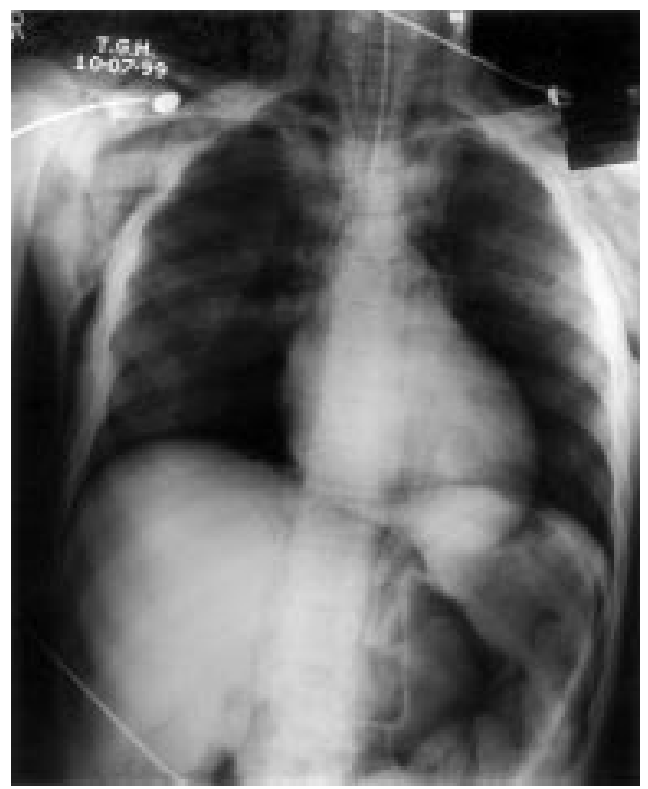

Figure 1 Chest radiography demonstrating extensive subcutaneous emphysema, mediastinal emphysema, bilateral small pneumothoraces (more obvious on the left) and double wall sign outlining the stomach suggestive of intraperitoneal free air. 


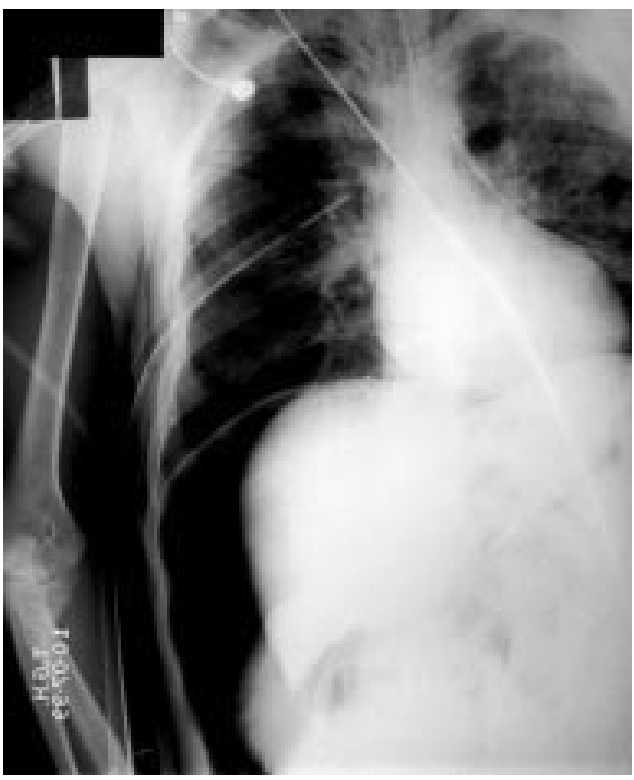

Figure 2 Lateral decubitus film confirming the presence of intraperitoneal free air.

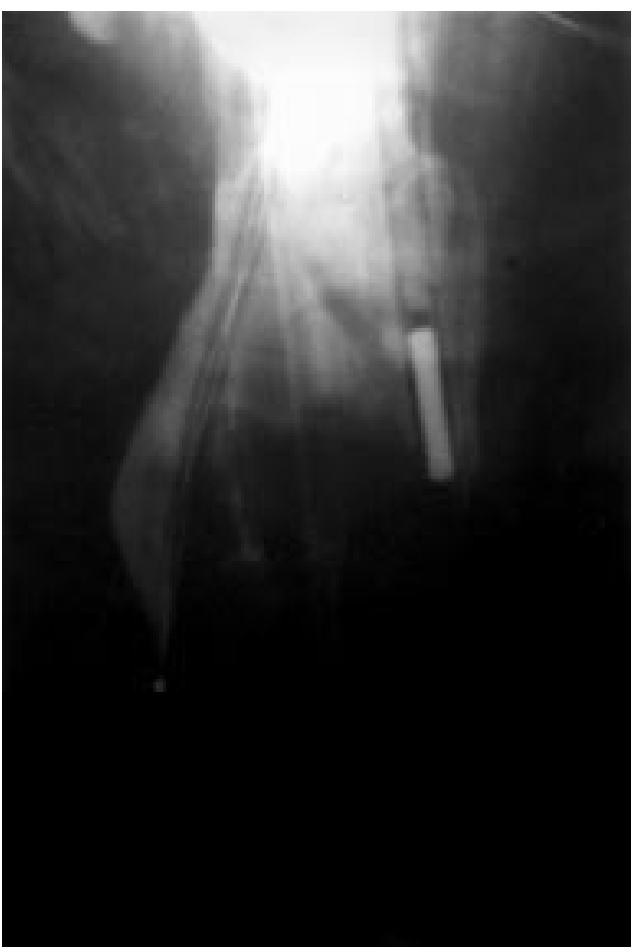

Figure 3 Gastrograffin contrast study showing a nasogastric tube in the left piriform fossa. Extravasation of contrast is seen in the hypopharynx to the right of midline

considered and an urgent water soluble contrast study performed. Oesophageal rupture was excluded but there was evidence of a perforation of the pharynx (fig 3). Computed tomography of the brain excluded an acute cerebral event as the cause of the collapse. The scan demonstrated widespread cerebral oedema. The patient was transferred to the intensive care unit for further management. High levels of amphetamines were later found on urine drug screening providing a possible cause for the initial collapse. The patient did not recover and after formal brain stem tests, was certified dead five days after admission.
Postmortem findings confirmed a small perforation in the posterior wall of the hypopharynx. There was no evidence of abdominal visceral perforation or diaphragmatic rupture.

\section{Discussion}

Hypopharyngeal perforation secondary to endotracheal intubation is a rare complication that is more likely to occur when performed as an emergency procedure outside a hospital environment or by less experienced personnel, or both. ${ }^{23}$ The commonest sites of rupture are the weak posterior pharyngeal wall and the pyriform fossae.

Pharyngo-oesophageal ruptures can present immediately with cervical surgical emphysema extending both proximally and distally. They may cause either unilateral or bilateral pneumothoraces, mediastinal emphysema resulting in respiratory distress. ${ }^{4} \mathrm{~A}$ sore throat, dysphagia, swelling and pyrexia occurring within days of intubation herald a late presentation of injury. Infection with retropharyngeal abscess formation if left unchecked can progress to pseudo-aneurysm formation of the common carotid artery, ${ }^{5}$ mediastinitis, pyopneumothorax, septic shock and death. ${ }^{56}$

The key to the management of these injuries is to have a high index of suspicion and to confirm the diagnosis and treat the injury as soon as possible. ${ }^{7}$

Chest and soft tissue radiographs of the neck are more reliable then clinical signs for assessing cervical and mediastinal emphysema. ${ }^{7}$ Endoscopy or water soluble contrast study is then recommended to confirm the diagnosis. In our ventilated patient we opted for initial radiological assessment as endoscopy in the presence of such gross surgical emphysema is difficult and can easily miss a mucosal defect. Water soluble studies are associated with a $25 \%-50 \%$ false negative rate therefore a negative water soluble study should be followed by barium study if clinically indicated. ${ }^{8}$ Computed tomography is a useful diagnostic tool in patients. It has the added advantage of localising the site of perforation helping the clinician to plan a suitable surgical approach. ${ }^{9} 10$

The management of these lesions remains controversial. The main argument lies in the decision to treat either conservatively or surgically. Relatively small perforations located in the cervical oesophagus and minor tears in the hypopharynx when recognised early can be treated conservatively with antibiotics and nasogastric drainage. ${ }^{4}$ Larger lesions in the hypopharynx and oesophagus require exploration, primary closure if possible and adequate drainage. ${ }^{11} \mathrm{~A}$ delay in operation and repair of the perforation greater than 12 hours after the injury is associated with a $56 \%$ mortality. ${ }^{12}$

We propose a possible sequence of events in our patient. The initial collapse was attributable to a cardiac arrest from an amphetamine overdose. It is thought that the hypopharyngeal perforation occurred during the first attempt at endotracheal intubation with air/ oxygen being forced into the surrounding soft tissue, mediastinum and thoracic cavity under pressure. Air/oxygen must have tracked down 
the mediastinum and escaped into the peritoneum giving rise to the pneumoperitoneum. A provisional diagnosis of pharyngooesophageal perforation was quickly made and confirmed with contrast studies. We opted for conservative management in this patient taking into consideration his prolonged cardiac arrest and poor neurological status.

In conclusion, endotracheal intubation is a common procedure and serious complications are uncommon. To make the diagnosis of a pharyngo-oesophageal perforation a high index of suspicion is required. The classic clinical presentation is swelling of the neck, surgical emphysema and respiratory distress. The presentation of a distended abdomen caused by a pneumoperitoneum resulting from a ruptured hypopharynx has not been previously described. Appropriate investigation quickly confirmed the diagnosis in our case but unfortunately because of other medical and neurological problems we were unable to save the patient.

Contributors

S A A Woodcock initiated and coordinated the writing up of the case, reviewed the case notes, carried out a MEDLINE search and co-wrote the paper. $\mathrm{H}$ Bird helped in the initial appraisal of the case. A K Siriwardena helped evaluate the relative importance of aspects of the case and co-wrote the paper. $S$ Ellenbogen discussed core ideas relating to the paper and is the guarantor.

1 Wolff A, Kuhn F, Ogura H. Pharyngeal-esophageal perforaions associated with rapid oral endotracheal intubation. Ann Otol Rhinol Laryngol 1972;81:258-61.

2 Lee T, Jordan J. Pyriform sinus perforation secondary to traumatic intubation in a difficult airway patient. F. Clin Anesth 1996;6:152-5.

3 Hawkins D, Seltzer D, Burnett T, et al. Endotracheal tube perfortion of the hypopharynx. West f Med 1974;120:2826.

4 Hirsch M, Abramowitz H, Shapira S, et al. Hypopharyngeal injury as a result of attempted endotracheal intubation. Radiology 1978;128:37-9.

5 Ooi G, Irwin M, Lam L, et al. An unusual complication of emergency tracheal intubation. Anaesthesia 1997;52:154-8.

6 Wengen D. Piriform fossa perforation during attempted tracheal intubation. Anaesthesia 1987;42:519-21.

7 Shockley WW, Tate JL, Stucker FJ. Management of perforaShockley WW, Tate JL, Stucker FJ. Management of perfora-
tions of the hypopharynx and cervical esophagus. Laryngoscope 1985;95:939-41.

8 Foley MJ, Ghahremani GG, Rogers LF. Reappraisal of contrast media used to detect upper gastrointestinal perforations. Radiology 1982;144:231-7.

9 Ward MP, Glazer HS, Heiken JP, et al. Traumatic perforation of the pyriform sinus: CT Demonstration. $f$ Comput Assist Tomogr 1985;9:982-4.

10 Schwartz, R Holliday, S Breda. CT diagnosis of pyriform sinus perforation. $\mathcal{F}$ Comput Assist Tomogr 1988;12:869-70.

11 Levine PA. Hypopharyngeal perforation: An untoward complication of endotracheal Intubation. Arch Otololaryngol 1980:106;578-80.

12 Dubost C, Kaswin D, Duranteau A, et al. Esophageal perforation during attempted endotracheal intubation. $\mathcal{F}$ Thorac Cardiovasc Surg 1979;78:44-5. 